\title{
L-band mode-locked femtosecond fiber laser with gigahertz repetition rate
}

\author{
JIAZHENG SONG, ${ }^{1,2}$ YUANSHAN LIU, ${ }^{1,3,{ }^{*}}$ AND JIANGUO ZHANG ${ }^{1,4,5}$ \\ ${ }^{1}$ State Key Laboratory of Transient Optics and Photonics, Xi'an Institute of Optics and Precision \\ Mechanics, Chinese Academy of Sciences, Xi'an 710119, China \\ ${ }^{2}$ University of Chinese Academy of Sciences, Beijing 100049, China \\ ${ }^{3}$ School of Electronics and Information@ @ OPTIMAL, Northwestern Polytechnical University, Xi'an \\ 710072, China \\ ${ }^{4}$ Division of Electrical and Electronic Engineering, School of Engineering, London South Bank \\ University, 103 Borough Road, London SE1 0AA, UK \\ 5zhangja@lsbu.ac.uk \\ *liuyuanshan@opt.ac.cn,
}

\begin{abstract}
We demonstrate an L-band passively mode-locked femtosecond fiber laser with a fundamental repetition rate of $1.013 \mathrm{GHz}$ based on semiconductor saturable-absorber mirror (SESAM). Compared with other reported L-band fiber lasers which use overlong Er-doped fiber (EDF), the laser here consists of $10 \mathrm{~cm}$ heavily doped fiber to increase the fundamental pulse repetition rate to gigahertz level. An output ratio as low as $0.2 \%$ is used to make the central wavelength up to $1.6 \mu \mathrm{m}$. The laser operates at the soliton regime with a $3 \mathrm{~dB}$ spectral bandwidth of $13.6 \mathrm{~nm}$, and a pulse duration of $229 \mathrm{fs}$.
\end{abstract}

(C) 2018 Optical Society of America under the terms of the OSA Open Access Publishing Agreement

\section{Introduction}

Fiber lasers at L-band $(1565 \mathrm{~nm}-1625 \mathrm{~nm})$ have attracted much attention due to their important role in many applications, including expanding the telecommunication window in optical communications $[1,2]$, reducing scattering and absorption to allow surgery in edematous corneas [3], increasing the transmission in brain tissue for biological imaging [4, 5]. In the past decades L-band fiber lasers have been widely investigated and dramatic improvements of L-band EDFLs have been made by researchers [6-29]. Wang et al. present an L-band ultrafast Er-doped all-fiber similariton laser with a sub-60 fs pulse duration, which is the shortest pulse duration ever reported to date [13]. A widely tunable multiple-soliton fiber laser is demonstrated by Meng et al., the central wavelength of which is continuously tunable over $75 \mathrm{~nm}$ range by controlling the intracavity loss and polarization controllers [14]. Later a dissipative soliton laser with a wider tuning range is reported [15], whose wavelength can be tuned from $1.6 \mu \mathrm{m}$ to $1.94 \mu \mathrm{m}$ via soliton self-frequency shift and chirped pulse amplifier, which make the laser a promising source for biomedical imaging. An efficient dissipative soliton mode-locked fiber laser is demonstrated by using nonlinear polarization rotation technology [17], which delivers pulses with single pulse energy of $6.15 \mathrm{~nJ}$. So far this is the highest single pulse energy reported for L-band passively mode-locked all-fiber laser. In general, for fiber lasers operating at L-band, the population inversion of the EDF should be limited to a low level $(30 \%-40 \%)$ to obtain a positive gain at $1.6 \mu \mathrm{m}$, while it is negative at $1.55 \mu \mathrm{m}$ [18]. The low population inversion could be achieved by various approaches, such as controlling the cavity losses [18-20], lengthening the EDF [21-23], using highly doped fibers [24-26], or cascading different types of the EDFs [12, 13]. However, no matter which approach is used, the length of the EDF is usually several meters, even tens of meters in some works. The overlong EDF make the laser difficult to work in stable mode-locking state and vulnerable to environmental influence, thus it is important to investigate the L-band fiber laser with much shorter EDF. With shorter EDF, the pulse repetition rate of the laser could be 
greatly increased, which would benefit a lot for the applications, such as increasing the imaging speed, widening the transmission capacity of optical communications and so on. However, shortening the EDF length causes the operating wavelength shift toward to 1.55 $\mu \mathrm{m}$, so heavily doped gain fiber and ultralow output ratio are required to make the wavelength stay at $1.6 \mu \mathrm{m}$ regime [12]. In the work reported by Byun et al. [28], an L-band gigahertz fiber laser with $1573 \mathrm{~nm}$ central wavelength is presented. Due to the high pump power up to $200 \mathrm{~mW}$, a piece of single mode fiber is used to protect the saturable Bragg reflector (SBR) from thermal damage. In contrast to other mode-locking techniques (e.g., single wall nanotube (SWNT) based saturable absorber [29], nonlinear polarization evolution [13, 30]), the passively mode-locked fiber laser using a SESAM as mode-locker is still more reliable due to the high threshold of thermal damage and compact, stable structure.

In this paper, we experimentally present a SESAM-based L-band passively mode-locked laser with gigahertz pulse repetition rate. To make the laser operate at long wavelength, we should decrease the cavity loss as low as possible. Due to the limited gain from the EDF, a SESAM with high saturation is used to make the laser easier to get self-starting. High saturation of SESAM usually accompanies with high non-saturable loss. Thus we decrease the cavity loss through lowering output ratio. A piece of EDF with $80 \mathrm{~dB} / \mathrm{m}$ gain coefficient is used as gain medium, and the output ratio is as low as $0.2 \%$, which make the laser operate at $1608.8 \mathrm{~nm}$. So far as we know this is the longest wavelength of a gigahertz level L-band fiber laser ever reported. Finally we present a fiber laser operating at soliton regime with a 3 $\mathrm{dB}$ spectral bandwidth of $13.6 \mathrm{~nm}$ and a pulse duration of $229 \mathrm{fs}$. The SNR of the RF spectrum is $81 \mathrm{~dB}$. The timing jitter integrated from $10 \mathrm{MHz}$ down to $200 \mathrm{~Hz}$ is $96.6 \mathrm{fs}$, which indicates a low-noise property. The long-term stability is also tested by continuously monitoring the output power. The result shows that the mode-locking state lasts for 10 hours with a relative rms noise of $0.14 \%$.

\section{Experimental Setup}

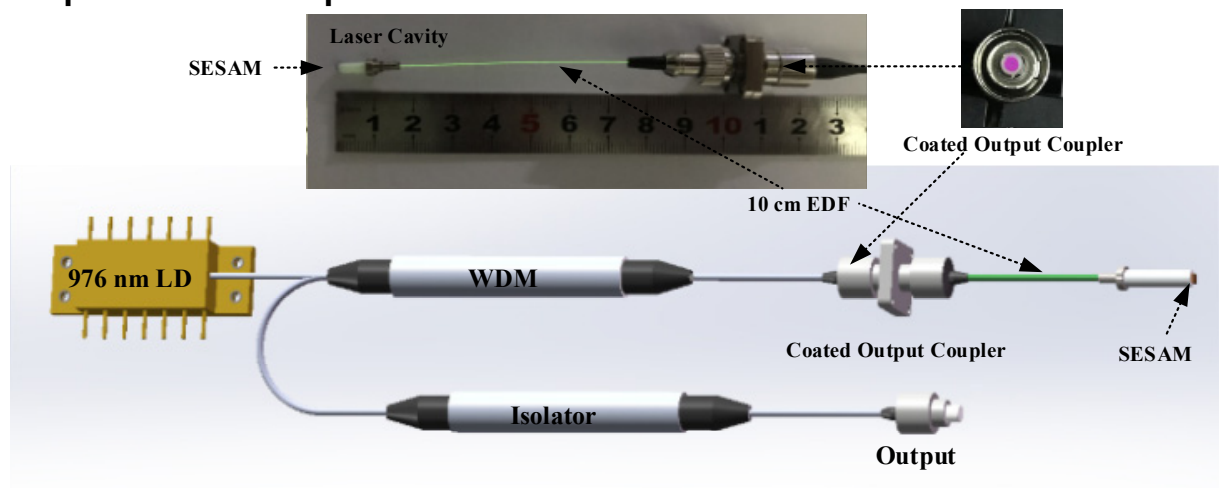

Fig. 1. Experimental setup of the L-band fiber laser.

The experimental setup of the L-band fiber laser is shown in Fig. 1. The laser cavity consists of a $10 \mathrm{~cm}$ EDF (Liekki Er80-8/125), a coated output coupler and a piece of SESAM. The heavily doped fiber provides the population inversion as gain medium. The gain fiber is secured with epoxy in $127 \mu \mathrm{m}$ inner diameter ceramic ferrules, both ends of which are flat polished. A coated fiber ferrule, as the output coupler, is connected to one end of the polished gain fiber in a mating sleeve. The output coupler shows a high reflectivity of $99.8 \%$ at L-band region, corresponding to a $0.2 \%$ output ratio for the $1.6 \mu \mathrm{m}$ optical signal, as well as a high transmittance of over $98 \%$ at $976 \mathrm{~nm}$ to reduce the pump loss. The other end of the gain fiber is butt-coupled to a SESAM (produced by Batop $\mathrm{GmbH}$ ), which is used as the mode-locker. Such a structure could promise that the spot area on the surface of the SESAM is small enough, so that the laser could self-start under the condition of low intracavity pulse energy. 
At the wavelength of $1608.8 \mathrm{~nm}$, the used EDF has an anomalous dispersion of $-26.4 \mathrm{fs}^{2} / \mathrm{mm}$ and the normal dispersion of the SESAM is $\sim 800 \mathrm{fs}^{2}$, thus the net intracavity dispersion is estimated to be $-4480 \mathrm{fs}^{2}$, which make the laser operate at soliton regime. The SESAM has $18 \%$ modulation depth, $30 \%$ absorbance and 5 ps recovery time.

The $976 \mathrm{~nm}$ pump light is coupled into the gain fiber through a wavelength division multiplexer (WDM) and the coated output coupler. The output pulses from the laser pass through an isolator to eliminate the influence of the unabsorbed pump light. The optical spectrum and pulse duration of the pulses is measured by an optical spectrum analyzer (AQ6370D, Yokogawa) and an autocorrelator (Autocorrelator PulseCheck, APE GmbH), respectively. The radio frequency $(\mathrm{RF})$ signal is detected by a high speed photodetector (UPD-15-IR2, Alphalas) and the RF spectrum and phase noise is directly measured by a signal analyzer (FSUP26, Rohde \& Schwarz).

\section{Results and Discussion}
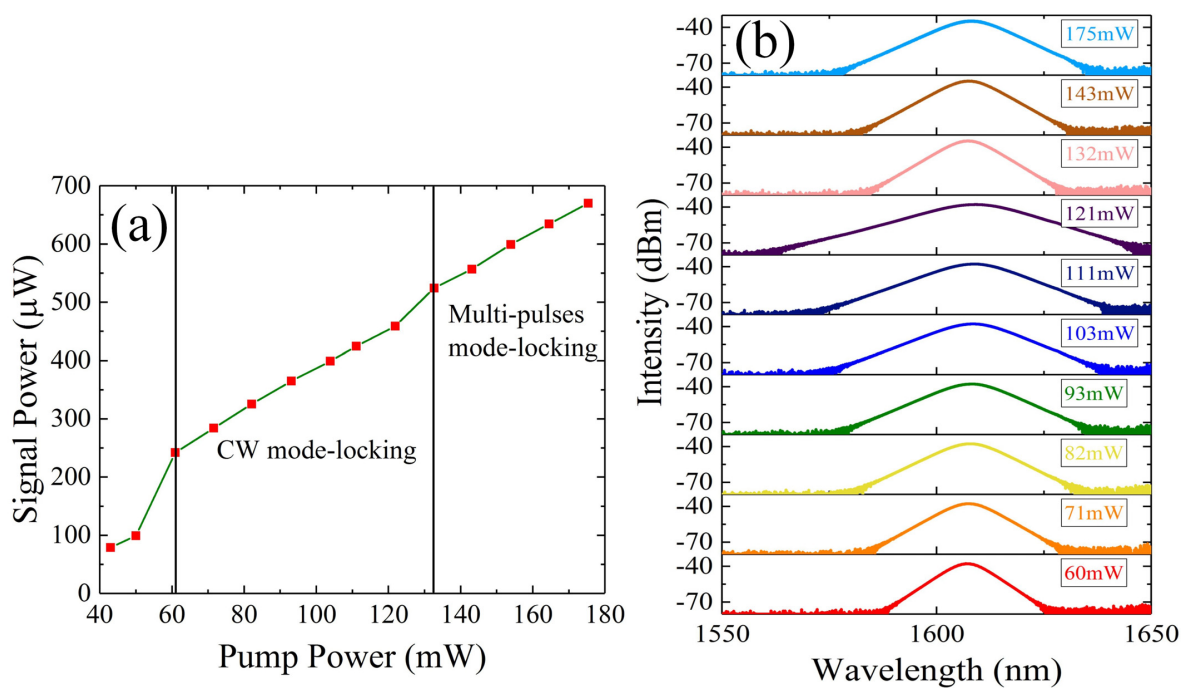

Fig. 2. (a) Signal power versus pump power. (b) Optical spectral versus pump power.

The dependence of the signal power and optical spectral on pump power is shown in Fig. 2. The laser realizes self-started mode-locking at a low threshold pump power of $60 \mathrm{~mW}$ with a $248 \mu \mathrm{W}$ output power, the optical spectrum of the mode-locked pulses centers at $1607.2 \mathrm{~nm}$ with a $3 \mathrm{~dB}$ spectral bandwidth of $6.4 \mathrm{~nm}$. With the increasing pump power, the signal power linearly grows and the central wavelength moves toward long wavelength with the $3 \mathrm{~dB}$ spectral bandwidth getting wider. While the pump power increases to $121 \mathrm{~mW}$, the output power is up to $470 \mu \mathrm{W}$ with a central wavelength of $1608.8 \mathrm{~nm}$ and a $3 \mathrm{~dB}$ spectral bandwidth of $13.6 \mathrm{~nm}$. With the further increasing of the pump power to $132 \mathrm{~mW}$, the $3 \mathrm{~dB}$ spectral bandwidth suddenly gets narrower from $13.6 \mathrm{~nm}$ to $6.84 \mathrm{~nm}$, indicating that the continuouswave $(\mathrm{CW})$ soliton mode-locking state turns to multi-pulsing mode-locking state. 

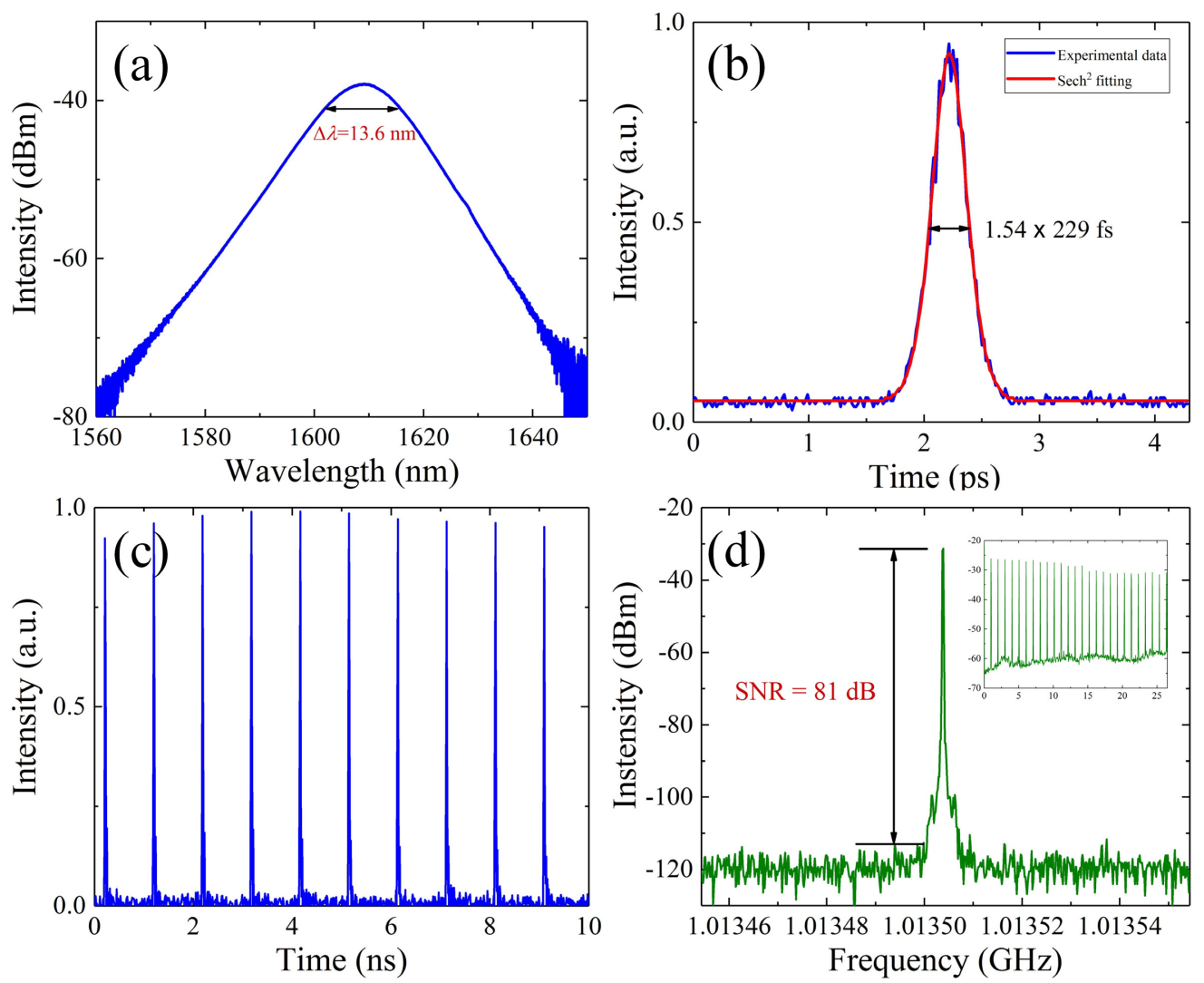

Fig. 3. (a) Optical spectrum from the laser. (b) Measured autocorrelation trace of the optical pulses (blue line) and $\mathrm{Sech}^{2}$ fitting curve (red line). (c) Oscilloscope trace of the pulses. (d) RF spectrum of the fundamental frequency with a resolution bandwidth of $100 \mathrm{~Hz}$. Inset: wide-span RF spectrum.

Figure 3(a) illustrates the optical spectrum of the output pulses measured at $121 \mathrm{~mW}$ pump power. The optical spectrum has a central wavelength of $1608.8 \mathrm{~nm}$ and a $3 \mathrm{~dB}$ spectral bandwidth of $13.6 \mathrm{~nm}$. Although the signal power is only $460 \mu \mathrm{W}$, due to the high sensitivity of $10^{-6} \mathrm{~W}^{2}$ of the photomultiplier detector in the autocorrelator, the intensity autocorrelation trace can be directly measured, as displayed in Fig. 3(b). The autocorrelation trace has a full width at half-maximum (FWHM) width of $353 \mathrm{fs}$, corresponding a temporal width of $229 \mathrm{fs}$, when a Sech pulse shape is assumed. The pulse width is slightly longer than the $199.8 \mathrm{fs}$ transform-limited duration, which indicates that the pulses are chirped. The oscilloscope trace of the pulses is depicted in Fig. 3(c), the corresponding temporal period is $987.5 \mathrm{ps}$. As shown in Fig. 3(d), the RF spectrum indicates a $1.013 \mathrm{GHz}$ fundamental pulse repetition rate, which is consistent with the temporal period in Fig. 3(c), and an $81 \mathrm{~dB}$ signal-to-noise ratio (SNR). The resolution bandwidth (RBW), video bandwidth (VBW) and span are $100 \mathrm{~Hz}, 100 \mathrm{~Hz}$ and $100 \mathrm{kHz}$, respectively. The inset to Fig. 3(d) shows the broad RF spectrum of the harmonics in a full span of $26.5 \mathrm{GHz}$. The mode-locked waveform of the oscilloscope trace and the clear intensity of the RF spectrum prove that the laser is operating at CW mode-locking state. 

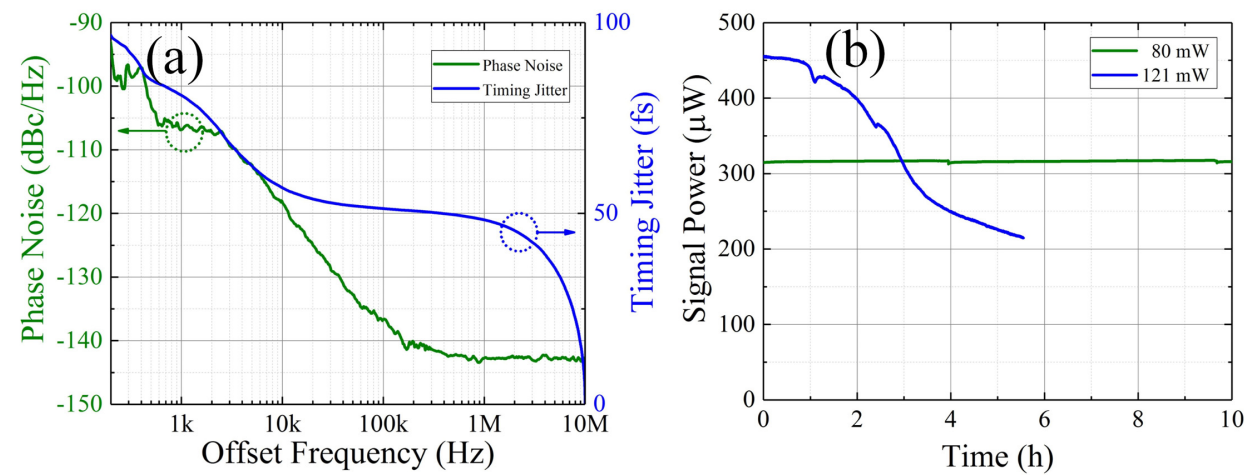

Fig. 4. (a) The SSB phase noise measured at the fundamental repetition rate of $1.013 \mathrm{GHz}$ and the corresponding integrated timing jitter. (b) Long-term measurement of the signal power with the pump power of (a) $80 \mathrm{~mW}$, (b) $121 \mathrm{~mW}$.

The single sideband (SSB) phase noise measured at the fundamental frequency of 1.013 $\mathrm{GHz}$ is displayed in Fig. 4(a). The phase noise at the low offset frequency is above -100 $\mathrm{dBc} / \mathrm{Hz}$, which is mainly contributed by the external technical noise (e.g., acoustic noise and power supply noise). Then it progressively decreases with a $-20 \mathrm{~dB} /$ decade slope while the offset frequency increasing to $100 \mathrm{kHz}$. The contribution to phase noise during this offset frequency range could be the amplified spontaneous emission (ASE) noise [31]. According to Eq. (1) in Ref. [32] and the parameters of the laser, we find the Gordon-Haus jitter is $16 \mathrm{~dB}$ lower than the direct ASE driven jitter, which indicates the direct ASE driven jitter contributes more to the phase noise than the Gordon-Haus jitter. Besides, the relativeintensity-noise-coupled jitters also affect the phase noise [31]. For the offset frequencies higher than $1 \mathrm{MHz}$, the phase noise remains around $-143 \mathrm{dBc} / \mathrm{Hz}$, which is limited by the shot-noise level of the measurement system. The timing jitter integrated from $10 \mathrm{MHz}$ progressively down to $200 \mathrm{~Hz}$ is $96.6 \mathrm{fs}$, given as the blue line in Fig. 4(a). Further optimization of the timing jitter could be achieved by suppressing the external technical noise, such as feedback control of the cavity length, the environmental temperature, and the power supply of the pump. The obtained timing jitter shows a low-noise property of the L-band laser.

Considering the $0.2 \%$ output ratio, the highest intracavity pulse energy of the $\mathrm{CW}$ modelocking pulses is calculated to be $232 \mathrm{pJ}$. The low threshold pump power and small single pulse energy make the SESAM avoid from thermal damage, therefore the operation of the laser remains stable for a long time. Setting the pump power to be $80 \mathrm{~mW}$, we monitor the output signal power for 10 hours at a sampling rate of one sample per second to test the longterm stability of the laser. The result of the power measurement is given in Fig. 4(b), a mean output power of $316.39 \mu \mathrm{W}$ with an rms noise of $0.4349 \mu \mathrm{W}$ gives a low relative rms noise of $0.14 \%$. Two power drops appear at $3.95 \mathrm{~h}$ and $9.6 \mathrm{~h}$, which might be caused by the vibration of the experimental platform, the fluctuations of pump power or some other occasional environmental changes. Besides, the long-term stability of the laser at $121 \mathrm{~mW}$ is also tested as the blue line in Fig. 4(b). Although the laser can keep CW mode-locking state for over 5 hours, the signal power decreases gradually to half the initial power, which results from the degradation of the SESAM. We think the degradation of the SESAM might be due to the twophoton absorption, which changes the modulation depth and the non-saturable loss of the SESAM $[33,34]$. Besides, the heat accumulation at the surface of the SESAM could also result in the degradation.

\section{Conclusion}

We demonstrate a SESAM-based L-band fiber laser with gigahertz fundamental repetition rate. The laser we present can operate at L-band without overlong EDF, optical filter, variable attenuator or complex structure such as cascading different types of EDFs. The simple, compact laser delivers $229 \mathrm{fs}$ optical pulses with a central wavelength of $1608.8 \mathrm{~nm}$ and a 3 
$\mathrm{dB}$ spectral bandwidth of $13.6 \mathrm{~nm}$. Compared with the reported gigahertz fiber laser [28], the central wavelength is extended by $35.8 \mathrm{~nm}$, which is comparable with the entire C-band wavelength range of $35 \mathrm{~nm}$. The extended wavelength range could benefit many applications, e.g. greatly increasing the optical communication capacity. The timing jitter integrated from $10 \mathrm{MHz}$ to $200 \mathrm{~Hz}$ is $96.6 \mathrm{fs}$, which exhibits low-noise performance. The laser achieves CW soliton mode-locking at a low pump power of $60 \mathrm{~mW}$ and turns to multi-pulsing modelocking state at $132 \mathrm{~mW}$. The low pump power and signal power keep the laser away from thermal damage and make it stable operate for 10 hours with a relative rms noise of $0.14 \%$. We believe the compact, stable structure and low-noise property of the laser guarantee it a great potential for practical applications.

\section{Funding}

This work was supported by National key R\&D Program of China (2016YFF0200700) and Natural Science Foundation of China (61875226).

\section{References}

1. A.K. Srivastava, S. Radic, C. Wolf, J. C. Centanni, J. W. Sulhoff, K. Kantor, and Y. Sun, "Ultradense WDM transmission in L-band," IEEE Photonics Technol. Lett. 12(11), 1570-1572 (2000).

2. M. Fernandez-Vallejo, D. Ardanaz, and M. López-Amo, "Optimization of the available spectrum of a WDM sensors network using a mode-locked laser," IEEE J. Lightwave Technol. 33(22), 4627-4631 (2015).

3. F. Morin, F. Druon, M. Hanna, and P. Georges, "Microjoule femtosecond fiber laser at $1.6 \mu \mathrm{m}$ for corneal surgery applications," Opt. Lett. 34(13), 1991-1993 (2009).

4. L. Shi, L. A. Sordillo, A. Rodríguez-Contreras, and R. Alfano, "Transmission in near-infrared optical windows for deep brain imaging," J. Biophotonics 9(1-2), 38-43 (2016).

5. Y. Ozeki, and D. Tashiro, "Fast wavelength-tunable picosecond pulses from a passively mode-locked Er fiber laser using a galvanometer-driven intracavity filter," Opt. Express 23(12), 15186-15184 (2015).

6. H. Chen, M. Leblanc, and G. W. Schinn, "Gain enhanced L-band optical fiber amplifiers and tunable fiber lasers with erbium-doped fibers," Opt. Communications 216, 119-125 (2003).

7. Z. G. Lu, J. R. Liu, P. J. Poole, S. Raymond, P. J. Barrios, D. Poitras, G. Pakulski, P. Grant, and D. Roy-Guay, "An L-band monolithic InAs/InP quantum dot mode-locked laser with femtosecond pulses," Opt. Express 17(16), 13609-13614 (2009).

8. J. Du, S. M. Zhang, H. F. Li, Y. C. Meng, X. L. Li, and Y. P. Hao, "L-band passively harmonic mode-locked fiber laser based on a graphene saturable absorber," Laser Phys. Lett. 9(12), 896-900 (2012).

9. Y. Meng, A. Niang, K. Guesmi, M. Salhi, and F. Sanchez, "1.6 $\mu \mathrm{m}$ high-order passive harmonic mode locking in a fiber laser based on graphene saturable absorber," Opt. Express 22(24), 29921-29926 (2014).

10. J. Zhao, Y. Wang, S. Ruan, P. Yan, H. Zhang, Y. H. Tsang, J. Yang, and G. Huang, "Three operation regimes with an L-band ultrafast fiber laser passively mode-locked by graphene oxide saturable absorber," J. Opt. Soc. Am. B 31(4), 716-722 (2014)

11. Y. Meng, G. Semaan, M. Salhi, A. Niang, K. Guesmi, Z. Luo, and F. Sanchez, "High power L-band modelocked fiber laser based on topological insulator saturable absorber," Opt. Express 23(18), 23053-23058 (2015).

12. Z. Wang, K. Qian, X. Fang, C. Gao, H. Luo, and L. Zhan, "Sub-90 fs dissipative-soliton Erbium-doped fiber lasers operating at $1.6 \mu \mathrm{m}$ band," Opt. Express 24(10), 10841-10846 (2016).

13. Z. Wang, L. Zhan, X. Fang, C. Gao, and K. Qian, "Generation of sub-60 fs similaritons at $1.6 \mu \mathrm{m}$ from an allfiber Er-doped laser," IEEE J. Lightwave Technol. 34(17) 4127-4133 (2016).

14. Y. Meng, M. Salhi, A. Niang, K. Guesmi, G. Semaan, and F. Sanchez, "Mode-locked Er: Yb-doped doubleclad fiber laser with 75-nm tuning range," Opt. Lett. 40(7), 1153-1156 (2015).

15. J. Kang, C. Kong, P. Feng, X. Wei, Z. Luo, E. Y. Lam, and K. K. Y. Wong, "Broadband high-energy all-fiber laser at 1.6 $\mu \mathrm{m}$," IEEE Photonics Technol. Lett. 30(4), 311-314 (2018).

16. K. Guesmi, F. Bahloul, G. Semaan, Y. Meng, M. Salhi, and F. Sanchez, "Widely tunable, narrow line width and low optical noise continuous-wave all fiber Er: Yb co-doped double-clad ring laser,” J. Opt. 19(1), 015501015506 (2017).

17. X. Wang, Y. Liu, Z. Wang, Z. Wang, and G. Yang, "L-Band Efficient Dissipative Soliton Erbium-Doped Fiber Laser With a Pulse Energy of $6.15 \mathrm{~nJ}$ and $3 \mathrm{~dB}$ Bandwidth of $47.8 \mathrm{~nm}$," IEEE J. Lightwave Technol. 37(4), 1168-1173 (2019)

18. K. Guesmi, Y. Meng, A. Niang, P. Mouchel, M. Salhi, F. Bahloul, R. Attia, and F. Sanchez, "1.6 4 m emission based on linear loss control in a Er:Yb doped double-clade fiber laser," Opt. Lett. 39(22), 6383-6386 (2014)

19. G. Lin, J. Chang, Y. Liao, and H. Lu, "L-band Erbium-doped fiber laser with coupling-ratio controlled wavelength tenability," Opt. Express 14(21), 9743-9749 (2006). 
20. G. Lin, and J. Chang, "Femtosecond mode-locked Erbium-doped fiber ring laser with intra-cavity loss controlled full L-band wavelength tenability," Opt. Express 15(1), 97-103 (2007).

21. X. Dong, P. Shum, N. Q. Ngo, C. C. Chan, B. Guan, and H. Tam, "Effects of active fiber length on the tenability of erbium-doped fiber ring lasers," Opt. Express 11(26), 3622-3627 (2003).

22. D. Yan, X. Li, S. Zhang, M. Han, H. Han, and Z. Yang, "L-band wavelength-tunable dissipative soliton fiber laser," Opt. Express 24(2), 739-748 (2016).

23. K. Y. Lau, E. K. Ng, M. H. Abu Bakar, A. F. Abas, M. T. Alresheedi, Z. Yusoff, M. and A. Mahdi, "Low threshold L-band mode-locked ultrafast fiber laser assisted by microfiber-based carbon nanotube saturable absorber," Opt. Communications 413, 249-254 (2018).

24. Z. Sun, A. G. Rozhin, F. Wang, V. Scardaci, W. I. Milne, I. H. White, F. Hennrich, and A. C. Ferrari, "L-band ultrafast fiber laser mode locked by carbon nanotubes," Appl. Phys. Lett. 93(6), 0.1114-061116 (2008).

25. H. Ahmad, Z. Z. Zulkifli, F. D. Muhammad, M. Z. Zulkifli, K. Thambiratnam, and S. W. Harun, "Mode-locked L-band bismuth-erbium fiber laser using carbon nanotubes," Appl. Phys. B 115(3), 407-412 (2014).

26. C. Yang, X. Guan, W. Lin, Q. Zhao, G. Tang, J. Gan, Q. Qian, Z. Feng, Z. Yang, and S. Xu, "Efficient $1.6 \mu$ m linearly-polarized single-frequency phosphate glass fiber laser," Opt. Express 25(23), 29078-29085 (2017).

27. H. Chen, H. Zhang, H. Zhang, X. Zhao, Y. Xu, Y. Zou, X. Ma, and L. Jin, "1.6- $\mu$ m-wavelength dissipative solitons mode-locked fiber laser based on the optimization of passive fibers distribution," Appl. Optics 57(24), 7070-7075 (2018).

28. H. Byun, M. Y. Sander, A. Motamedi, H. Shen, G. S. Petrich, L. A. Kolodziejski, E. P. Ippen, and F. X. Kärtner, "Compact, stable $1 \mathrm{GHz}$ femtosecond Er-doped fiber lasers," Appl. Opt. 49(29), 5577-5582 (2010).

29. W. S. Kwon, H. Lee, J. H. Kim, J. Choi, K. Kim, and S. Kim, "Ultrashort stretched-pulse L-band laser using carbon-nanotube saturable absorber," Opt. Express 23(6), 7779-7785 (2015).

30. J. L. Luo, L. Li, Y. Q. Ge, X. X. Jin, D. Y. Tang, D. Y. Shen, S. M. Zhang, and L. M. Zhao, " $L-B a n d$ Femtosecond Fiber Laser Mode Locked by Nonlinear Polarization Rotation," IEEE Photonics Technol. Lett. 26(24), 2438-2441 (2014).

31. J. Kim, and Y. Song, "Ultralow-noise mode-locked fiber lasers and frequency combs: principles, status, and applications," Adv. Opt. Photon. 8(3), 465-640 (2016).

32. R. Paschotta, "Timing jitter and phase noise of mode-locked fiber lasers," Opt. Express, 18(5), 5041-5054 (2010)

33. E. R. Thoen, E. M. Koontz, M. Joschko, P. Langlois, T. R. Schibli, F. X. Kärtner, E. P. Ippen, and L. A. Kolodziejski, "Two-photon absorption in semiconductor saturable absorber mirrors," Appl. Phys. Lett. 74(26), 3927-3939 (1999)

34. T. R. Schibli, E. R. Thoen, F. X. Kärtner, and E. P. Ippen, "Suppression of Q-switched mode locking and breakup into multiple pulses by inverse saturable absorption” Appl. Phys. B 70(Suppl. 1), S41-S49 (2000). 